\title{
Educación integral comunitaria y afroepistemología en Cuyagua, Venezuela
}

\author{
Community integral education and afroepistemology in Cuyagua, Venezuela
}

\begin{abstract}
Alberto Preciado
https://orcid.org/0000-0001-9470-0988

Sociólogo, Doctor en Ciencias para el Desarrollo Estratégico de la Universidad Bolivariana de Venezuela. Docente Universidad de Antioquia, Medellín, Colombia.

Correo-e: doprecia.alberto@gmail.com
\end{abstract}

Fecha de recepción: 15 de enero de 2017

Fecha de aprobación: 21 de agosto de 2017

Cómo citar este artículo/ to reference this article

Preciado, A. (2017). Educación integral comunitaria y afroepistemología en Cuyagua, Venezuela. Derecho $Y$

Realidad, 15(30): e28175-1. Recuperado de

https://revistas.uptc.edu.co/index.php/derecho_realidad/article/view/9248

Resumen: la motivación académica es un acercamiento a la construcción de intersubjetividades con las(os) cimarronas(es) de la Red de Organizaciones Afrovenezolanas, en adelante ROA, a partir de la experiencia educativa del Centro de Educación Integral Comunitaria (CEICO) que vivieron en el pueblo de Cuyagua, municipio Costa de Oro del Estado de Aragua, en la República Bolivariana de Venezuela. Esta experiencia educativa, vista desde la orientación cualitativa, sirvió de base para abordar una reflexión teórico-metodológica en relación con la presencia de la afroepistemología y la afroepistemetódica -categoría construida por el profesor afrovenezolano Jesús "Chucho" García y entendida como epistemología decolonial, desde la perspectiva Modernidad/Colonialidad/Decolonialidad-, además, los procesos históricos, vinculados en las luchas por la educación en las comunidades afrodescendientes del país, desde la perspectiva del maestro Luis Bigott. Asimismo, el trabajo de campo a través del método de la investigación, como un estudio de caso cualitativo, cercano a la investigación colaborativa o cooperativa, el cual permitió relacionar las categorías emergentes de educación integral comunitaria, maestras(os) del pueblo, pedagogía de la oralidad, racismo epistémico y pedagogía del cimarronaje, visibilizando los aportes de las(os) afrodescendientes, lo cual permitió concluir que es necesario construir en el país una educación crítica y política, ligada a las necesidades y contextos de las poblaciones a las cuales va dirigida.

Derecho real

Julio - Diciembre 2017 
Palabras clave: educación integral, comunidad, afroepistemología, afroepistemetódica, modernidad, colonialidad, decolonialidad.

\begin{abstract}
The academic motivation was to approach the construction of intersubjectivities with the cimarrones of the Network of Afro-Venezuelan Organizations, from now on ROA, based on the educational experience of the Center for Community Integral Education (CEICO) who lived in the town of Cuyagua, Costa de Oro municipality of the Aragua state in the Bolivarian Republic of Venezuela. This educational experience, seen from the qualitative orientation, served as the basis to approach a theoretical-methodological reflection in relation to the presence of Afroepistemology and Afro-Epistemetodics-a category constructed by the Afro-Venezuelan professor Jesús "Chucho" García and understood as decolonial epistemology from the perspective of Modernity/Coloniality/Decoloniality-, in addition, the historical processes linked in the struggles for education in Afro-descendant communities of the country from the perspective of the teacher Luis Bigott. Likewise, the field work through the research method as a qualitative case study close to collaborative or cooperative research, allowed to relate the emerging categories of community integral education, village teachers, pedagogy of orality, epistemic racism and pedagogy of the cimarronaje, making visible the contributions of the afrodescendants, which allowed to conclude that it is necessary to build in the country a critical and political education, linked to the needs and contexts of the populations to which it is directed.
\end{abstract}

Key words: community, integral education, afroepistemology, afroepistemetodics, modernity, coloniality, decoloniality.

\title{
Introducción
}

Este artículo está orientado a presentar algunas reflexiones ontológico-epistemológicas, metodológicas y axiológicas, desde la perspectiva cualitativa, en relación con la educación integral comunitaria y la afroepistemología, a partir de la recuperación de una experiencia vivida por sus habitantes en el pueblo de Cuyagua, municipio Costa de Oro del Estado de Aragua de la República Bolivariana de Venezuela, entre los años 2002 y 2007. El objetivo general fue estudiar, desde la perspectiva teórica, los asuntos: Modernidad/Colonialidad/Decolonialidad, la experiencia en educación integral comunitaria, y su afroepistemología y afroepistemetódica, en relación con la Red de Organizaciones Afrovenezolanas (ROA) en Cuyagua.

El acceso al conocimiento de esta experiencia se dio gracias a la construcción de intersubjetividades con la gente de la ROA desde el año 2011, no obstante, como referente histórico, desde la propia experiencia y según lo dialogado con los actores, estos participaron allí 
desde el Segundo Encuentro Nacional de Mujeres Afrovenezolanas, realizado entre el 24 y el 26 de mayo del año 2002, en la Posada Casa Grande de Cuyagua, precisamente una de las principales promotoras para la creación del Centro Educativo Integral Comunitario Cuyagua (CEICO-Cuyagua).

Así, en una de las primeras entrevistas informales, realizada en diciembre del año 2011 en Cuyagua, para comprender el origen y sentido del CEICO, una de las profesoras, Emilsa Rivero, que estuvo en sus inicios y quien aún trabajó en la Unidad Educativa expresó lo siguiente:

El CEICO llegó como un programa de innovación a esta comunidad, como un centro comunitario integral donde se pone de manifiesto todo el contexto local, toda la trayectoria como la historia, la gastronomía, las manifestaciones culturales, la forma de vida que tenemos aquí en la comunidad. El CEICO nace de una propuesta que hizo la comunidad por haber sufrido por tantos años los muchachos jóvenes que teníamos que ir a estudiar a la comunidad de Ocumare de la Costa y era muy costoso para las personas aquí de escasos recursos, este, cómo mantener a un niño fuera de la comunidad y tenía que viajar a diario, todos los días, donde el transporte era bastante pesado, donde no tenía para costear los gastos de transporte, de comida, de útiles escolares, bueno y nació según la propuesta de una reunión con el movimiento afrovenezolano, dentro de la comunidad.

La profesora Rivero contó su vivencia porque ella hizo parte de una de esas generaciones que no tuvo con un liceo para terminar el bachillerato en su propio pueblo y por eso tuvo que caminar diariamente hasta tres horas o más, una distancia de 30 kilómetros, para poder llegar a Ocumare de la Costa y lograr su sueño de ser bachiller, ello en una región donde las oportunidades educativas eran bastante limitadas, pues existía un solo liceo y además con una infraestructura que hoy en día no presenta muy buenas condiciones.

De acuerdo con Martínez de Crespo (2003), la propuesta del CEICO tiene como principal característica favorecer una construcción y un desarrollo curricular, el cual se concreta a través de:

- La investigación, formación, organización y comunicación permanente de los actores del hecho educativo comunitario.

- La reconstrucción de la memoria histórica local, regional, nacional e internacional.

- El impulso y reconocimiento de la identidad local, regional, nacional e internacional.

- La promoción y desarrollo del poder local.

- La promoción de un modelo de desarrollo endógeno con base social.

Las preguntas de investigación estuvieron relacionadas con la concepción de afroepistemología y afroepistemetódica, la cual emergió en el inicio de la investigación, en una interacción teórica y empírica que, a su vez, combinó la coherencia teórico-metodológica del estudio, con su 
pertinencia para la comunidad como propuesta socioeducativa. En este sentido se hizo referencia a las interrogantes:

- ¿Cómo percibieron las(os) habitantes de Cuyagua-Venezuela, el racismo en cuanto a su derecho a una educación adecuada con su contexto social, económico, político, ambiental, cultural y territorial?

- ¿Cómo se vivieron las concepciones de la afroepistemología y afroepistemetódica dentro de la experiencia educativa del CEICO en Cuyagua-Venezuela?

- ¿Cómo podemos apropiarnos, mediante la ROA, de las concepciones de la epistemología del Sur, como una epistemología que reconoce la afroepistemología y afroepistemetódica, y con ella la lucha por la visibilización y el autoreconocimiento afrodescendiente en Venezuela?

Estas tres preguntas surgieron al establecer las relaciones entre la reivindicación de la afroepistemología y afroepistemetódica, construida por el educador afrovenezolano Jesús "Chucho" García, como un esfuerzo compartido y logrado en la ROA, una experiencia concreta que vivieron en el pueblo de Cuyagua, con la creación del CEICO que, a su vez, se puede enmarcar en el planteamiento de la teoría de la epistemología del Sur, sustentada por el sociólogo portugués Boaventura de Sousa Santos.

El trabajo se dividió en las siguientes secciones: sustento metodológico de la investigación, método y las técnicas como construcción instrumental, la perspectiva teórica, la problemática del racismo epistémico, la Modernidad/Colonialidad/Decolonialidad como nueva visión sociocrítica decolonial, y la Afroepistemología y la epistemología del Sur y sus conclusiones.

\section{Metodología}

Combinado con la perspectiva teórica, la construcción del sustento metodológico, mediante unos elementos discursivos, donde lo humano y observado desde las cotidianidades y lo técnico instrumental de la investigación, fueron surgiendo en un proceso donde el contexto sociocultural cobró fuerza y debido a la aceptación de investigar con las y los habitantes del pueblo de Cuyagua en la costa aragüeña, en el cual la receptividad de esa población hizo al autor ser partícipe del doble rol: investigador-actor. 
Esto ha sido parte de las ideas que se han plasmado desde la coherencia teórica que exige el diseño cualitativo entre metodología, método y técnicas de recolección de información que, según Ibáñez (1990), es el camino para producir nuevos conocimientos, en este caso, partiendo de la perspectiva teórica, la cual resultó acorde con el mismo proceso de investigación, es decir, Modernidad/Colonialidad/Decolonialidad, como se ha venido argumentando, en un recorrido ontológico-epistemológico. Cabe destacar igualmente que el rumbo de la investigación ha ido cambiando paulatinamente, en relación con el proyecto inicial, orientado bajo la mirada de investigación social emergente sociocrítica. Además, ahora el trabajo de campo sigue un proceso de construcción del método, algo diferente al de la Investigación-Acción-Participativa (IAP) en sentido estricto, como lo plantearon inicialmente sus autores más allegados, entre ellos Fals Borda y González (1991).

\section{Momentos de la investigación}

La investigación se caracterizó por la producción permanente y articulada de conocimientos, construida en el tiempo, mediante un proceso intersubjetivo, definiéndose en conjunto a los actores, de donde emergió su temática y problemática. No obstante, este es un proceso que evita lo apriorístico y lo unilateral en el sentido de que pasó por la generación progresiva de la relación de confianza y conocimiento entre el investigador y la gente de la ROA además, como se ha podido interpretar desde un principio, tiene una postura política y teórica sumamente críticas ante la academia tradicional, la cual las(os) ha visto solo como un objeto de estudio y no como iguales, con conocimientos significativos que emergen y dan cuenta de una realidad social interdependiente ante los saberes impuestos por el capitalismo neoliberal.

Por esta razón, es sumamente complejo seguir unos procedimientos guiados por otros predeterminados y, en vez de ello, aparece un proceso de construcción de los momentos que, si bien están separados, es solo como estrategia de presentación, pero que se han dado en un proceso de avance casi simultáneamente por su naturaleza más bien holística que lineal. Sin embargo, se encuentra en Valles (1999), basado en Morse, el planteamiento de seis fases para el diseño y realización de un estudio cualitativo, diferentes en su forma, pero que puedan decirse que la idea de fondo presenta similitud en cuanto a la búsqueda de reflexividad del investigador. Estas fases son: reflexión, planeamiento, entrada, recogida productiva y análisis preliminar, salida del campo y análisis intenso y, finalmente, escritura.

Sin reñir con lo anterior, también en la investigación fueron surgiendo siete momentos distintos de manera espontánea, sin separaciones espacio-temporales, los cuales pudieran dañar la riqueza investigativa, pero que ahora, obedeciendo a la sistematización de un proceso cualitativo emergente, es posible establecer como: 
a) Acercamiento a la ROA. Fue la llegada a la primera reunión con miembros de la ROA para presentar el interés particular de construir con ellas(os) el proyecto de tesis doctoral desde la visión cualitativa, a partir de una experiencia relevante para la Red.

b) Construcción de la temática y la problemática de estudio. Implicó diálogo permanente con la gente de la ROA, reuniones, discusiones, lectura de documentos sobre el CEICO y preguntas constantes para tratar de comprender, en el proceso, el objeto de estudio y la problemática a investigar.

c) Identificación de la perspectiva teórica que orientaría la investigación. Se abordó, desde la perspectiva Modernidad/Colonialidad/Decolonialidad, muy apropiada con la temática y problemática de estudio: educación integral comunitaria, afroepistemología y racismo epistémico.

d) Trabajo de campo. No solo se realizó en las visitas al pueblo de Cuyagua, en Venezuela, para realizar las primeras observaciones participantes, conversaciones y entrevistas informales, las cuales fueron profundizadas de forma cualitativa, así como diferentes espacios y escenarios de interacción de la ROA.

e) Interpretación de la información cualitativa. Con el propósito de profundizar en la información escrita y luego de realizar las entrevistas, fue necesario transcribirlas y extraer de las mismas la información directamente relacionada con el objeto de estudio, orientada hacia las preguntas, los objetivos y los conceptos centrales del estudio. Para la categorización se utilizó un sencillo programa de computación en inglés llamado Weft$Q D A$ versión 1.0.1, software libre para el análisis de datos cualitativos creado por Alex Fenton, un antropólogo social nacido en Inglaterra. Este programa permitió organizar un árbol de categorías y subcategorías de análisis, a partir de las transcripciones de las entrevistas, notas de campo producto de las observaciones participantes y los distintos documentos sobre el CEICO, afroepistemología y afroepistemetódica, afrovenezolanidad y todo lo que se consiguió, relacionado con la temática y la problemática de la investigación.

f) Reflexión crítica. Como cierre del trabajo de campo, la reflexión crítica fue indispensable para conceptualizar lo que significó la educación integral comunitaria y la afroepistemología, a partir del caso de la Investigación-Acción con la ROA en Cuyagua Venezuela.

g) Socialización y devolución en el pueblo de Cuyagua. Como parte de los compromisos éticos que genera la investigación cualitativa, se socializó la investigación en el marco de un evento comunitario. 


\section{El método y las técnicas como construcción instrumental}

En la misma línea, con todo lo anterior, puede decirse que el método empleado en la construcción de la investigación, como estudio de caso cualitativo, se aproximó a la investigación-acción cooperativa o colaborativa, pues se trató de una investigación realizada con profesoras(es)investigadoras(es) de la ROA, a partir de su propia experiencia educativa, vivida en el contexto de estudio en Cuyagua y en la cotidianidad que permitió interactuar con sus demás pobladores.

Tal como se ha venido señalando, los momentos en el proceso investigativo surgieron desde una visión ontológica-epistemológica, la cual permitió conocer, en líneas generales, la problemática del racismo epistémico, en una comunidad donde sus habitantes se han mantenido en constante lucha por defender su derecho a la educación integral, desde su cultura ancestral y de no permitir ser objetos de investigación. Este compromiso trae, como consecuencia, la selección instrumental que permitió el acercamiento a la gente, de la manera más humana posible. En estos términos, la experiencia en la comunidad de Cuyagua se inspiró especialmente en la conversación espontánea, González (2007), como instrumento de investigación o técnica que, junto a las entrevistas cualitativas en profundidad y a la observación participante, produjeron la información necesaria para comprender e interpretar su cultura socioeducativa.

La conversación informal o espontánea fue la principal forma de conocer la experiencia educativa del CEICO y tuvo mucho sentido por el valor que esta tiene para las comunidades afrodescendientes, la cual hace parte de su pedagogía de la oralidad y, aunque existen documentos escritos sobre el CEICO y algunos audiovisuales a los cuales hubo acceso, se privilegió la palabra sobre lo escrito.

Las conversaciones se llevaron a cabo fundamentalmente con profesoras(es) que trabajaron en la fundamentación y en la puesta en marcha del CEICO, también con otras personas de la comunidad entre ellas ex-estudiantes ya egresados, padres, madres y representantes que podían dar cuenta de lo que allí aprendieron.

La observación directa y participante, relacionada con las conversaciones con la gente, por supuesto fue fundamental, sobre todo en el pueblo de Cuyagua, porque se fue hasta el lugar y se conoció su contexto histórico, geográfico, económico, educativo, social y cultural, en el cual se vivió la experiencia educativa.

Las entrevistas cualitativas en profundidad, las cuales iniciaron como conversaciones y entrevistas informales y fueron el medio por el cual se pudo conocer más a fondo las motivaciones, dificultades y avances de la propuesta educativa y que básicamente estuvieron dirigidas a dos tipos de informantes: por un lado, las(os) conocedoras(es) de todo lo que fue el CEICO y, por otro, los académicos con amplia experiencia en el tema educativo de las 
comunidades afrodescendientes en el país y de la categoría afroepistemología y afroepistemetódica.

\section{La perspectiva teórica}

En un primer momento de reflexión sobre el tema de estudio se presentaron infinidad de ideas, entre ellas la postura ontológica-epistemológica que, de inmediato, condujo a las distintas orientaciones metodológicas interpretativas, asumiendo desde un principio la importancia del ser humano con un universo de conocimientos, el cual se refleja espontáneamente por sus vivencias cotidianas. Esto permitió realizar la investigación desde la perspectiva cualitativa, lo cual implicó un largo proceso de comprensión e interpretación de intersubjetividades en el contexto social que conformó la ROA. Fue un estudio que se definió alejado de lo apriorístico, en el sentido de que intervenían las visiones compartidas, no solo desde la visión del investigador, ni con la pretensión de un camino claro y preciso desde el comienzo sino que, por el contrario, prevaleció la complejidad, dependiendo del tiempo, de los ritmos, de los procesos y de las múltiples opiniones de las(os) otras(o) y exigía tratar de comprender sus experiencias desde su propio discurso, sin limitarse a la hermenéutica que, sin duda, cambiaría en parte el sentido de lo que fue la vivencia de estos sujetos.

El tema de estudio estuvo relacionado con la experiencia educativa del Centro de Educación Integral Comunitaria, vivida por la comunidad en el pueblo de Cuyagua, municipio Costa de Oro del Estado de Aragua pero que, en el caso particular, no generó la oportunidad de vivir directamente. En este sentido, se retomó -con su consentimiento- el producto del esfuerzo realizado por las(os) docentes-investigadoras(es), el cual consistió en la construcción de los escenarios de Investigación-Acción en el mes abril del año 2002; luego, durante el año 2003, en la constitución del CEICO, institución que funcionó desde el 26 de abril del año 2003 hasta el 27 de julio del año 2007 cuando pasó a llamarse Unidad Educativa Nacional Cuyagua, según el oficio emanado de la Zona Educativa de Aragua.

Gran parte de esta idea, compartida para la construcción de la investigación cualitativa en la tesis doctoral del autor, tuvo mucho que ver con las motivaciones surgidas a partir de la formación profesional en sociología obtenida por el autor en la Universidad de Antioquia, en Medellín, Colombia, donde siempre se sintió mucho interés en la investigación cualitativa de procesos sociales relacionados con los temas comunitarios, además de la necesidad de vivirlos como actor y de sentir directamente los conflictos que allí se presentan.

Desde esta perspectiva, un grupo de profesoras(es) investigadoras(es) de la ROA, participantes en ésta investigación, planteó que la academia tradicional, que es fundamentalmente eurocéntrica y positivista, no reconoce la afroepistemología y afroepistemetódica esto es, que no reconoce sus prácticas y conocimientos, lo cual constituye un epistemicidio. En este sentido, se puede afirmar 
que con las(os) afrodescendientes ocurrió lo mismo que con los pueblos indígenas desde 1492, reforzado con la conquista y colonización de América por parte de Europa. Igualmente, se trata de un onticidio (negación de la existencia del otro), el cual incluyó la negación de su capacidad lingüística.

En palabras del profesor puertorriqueño Ramón Grosfoguel, de la Universidad de Berkeley (California) y miembro del Grupo Modernidad/Colonialidad, se produjo contra las(os) afrodescendientes un racismo epistemológico, (2011), es decir, la inferiorización de sus saberes y formas de producir conocimientos. Por ello, no se reconoció a la afroepistemología y afroepistemetódica como legítimas, lo que sí se hizo con el positivismo, paradigma de investigación social hegemónico en Occidente e impuesto a Nuestra América y hoy día defendido por muchas instituciones que, de manera cíclica, presentan su visión objetivista la cual finalmente termina conociendo lo externo y medible del sujeto investigado.

En síntesis, el racismo en Venezuela y en el mundo pareciera un problema histórico, social, económico, político, cultural y territorial que va más allá de la discriminación racial, pero que tiene dimensiones que incluyen especialmente lo epistemológico como tema de esta investigación, además, que se recrudece cuando se reivindican las otras epistemologías, por ejemplo, la afroepistemología y las epistemologías del Sur.

\section{La problemática del racismo epistémico}

Como se mencionó anteriormente, el desconocimiento del otro lleva al desconocimiento de su historia, de sus prácticas y conocimientos. Por ende, la negación se genera en todos los planos: en el lenguaje, en el simbólico y, más grave aún, en el epistemológico.

Directamente asociado con el problema del racismo epistémico, Quijano (2009), peruano, plantea la noción de colonialidad del poder, entendida como:

Un patrón de dominación/explotación cuyo principio organizador reside en la clasificación etno-racial de las poblaciones del mundo. La idea de raza viene a entenderse como principio organizador de la división internacional del trabajo y de la distinción de los Estados entre centros, periferias y semi-periferias en el sistema interestatal global. "Raza" no se refiere exclusivamente a diferencias de color, sino a una clasificación construida a partir de jerarquías sexuales, de género, lingüísticas, epistémicas donde el elemento articulador y clasificador es la jerarquía etno-racial global entre Occidentales (como el lado superior) y no Occidentales (como el lado inferior). (p. 39.) 
Esta noción de colonialidad se enmarca en la modernidad occidental que, a su vez, se compone de la colonialidad del poder, del saber y del ser. La del saber porque la epistemología eurocéntrica y sus procesos de producción de conocimiento han reproducido un sistema donde predomina una epistemología racista y, por otro lado, la del ser debido a referencia a los grupos sociales invisibilizados desde esa concepción hegemónica moderna, eurocéntrica y racista.

Otros autores como Herrera (2009), venezolano, sostienen que aunque se lo niegue, el “...racismo ha constituido un elemento fundamental de la ideología y de las prácticas hegemónicas en Venezuela desde la Conquista hasta nuestros días" (p. 13). Este mismo autor cita a van Dijk, reconocido lingüista holandés, quien sostiene la existencia de un racismo académico, según él, tan antiguo como el racismo mismo.

De acuerdo con el Foro Social Mundial Temático Venezuela, se afirma:

que el racismo implica destrucción, desprecio, invisibilidad, distorsión de la imagen propia, muerte de las personas mismas, muerte de la memoria colectiva, muerte de la cultura y del ecosistema natural, integración al capitalismo e imitación de su modelo de vida, neocolonialismo, despojo territorial, explotación del trabajo humano y de la naturaleza, estigmatización, entre muchas problemáticas que es necesario visibilizar, denunciar, y contribuir como movimientos, comunidades y personas a resolver.

Esta visión se socializó en la convocatoria del Centro de Estudios Latinoamericanos "Rómulo Gallegos" (CELARG) para el Primer Encuentro "Visibilizando a los pueblos indígenas y afrodescendientes: por una estrategia para la erradicación de la discriminación, el neocolonialismo y el racismo en Venezuela", realizado el 22 de febrero del año 2013 en Caracas.

Desafortunadamente el racismo continúa vigente en el país, a pesar de los enormes esfuerzos que hacen las organizaciones sociales y políticas, víctimas del mismo y el propio gobierno bolivariano por erradicarlo y aunque cuentan con una Ley Orgánica contra la Discriminación Racial, en algunos casos y por el contrario, el racismo se ha afianzado como una práctica social, política y económica desde el neocolonialismo.

Por ello, es muy importante la realización de este tipo de encuentros, donde se visibilice, denuncie y se propongan acciones para enfrentarlo articuladamente, donde se busque comprender cómo hay diversas dimensiones fundamentadas en cosmovisiones, ideologías, teorías y prácticas de grupos sociales y de pueblos, las cuales se consideran a sí mismos como razas superiores, con derecho a dominar a los otros, a partir de su origen étnico y cultural, desde su procedencia y desde la pureza de sangre y que han llegado a convertirse en leyes y paradigmas hegemónicos en la modernidad occidental, en detrimento de la historia, los conocimientos y las prácticas de otros pueblos y grupos sociales, en especial los de origen subsahariano. 
Valga precisar que no se trata de un estudio sobre el racismo en sí, sino de intentar comprender cómo y por qué las prácticas y conocimientos de unos pueblos y grupos sociales son considerados como superiores, mientras otras, en cambio, son invisibilizadas (inferiorizadas) históricamente.

En ese sentido, como investigador y, al mismo tiempo, con el rol de actor, junto a los demás actores en este contexto geopolítico y educativo, en conjunto, las(os) miembros de la comunidad de Cuyagua, se han podido llevar a cabo las primeras conversaciones, las cuales se sintetizan en proponer con énfasis la integración de la afroepistemología y la afroepistemetódica en el currículo, desde la educación básica hasta la educación superior, debido a que los aportes de las(os) africanas(os), arrancadas(os) de sus tierras y esclavizadas(os) para el trabajo forzado en América y sus descendientes nacidas(os) en esta tierra, con sus propias prácticas y sus culturas, deberían ser reconocidos como parte fundamental de la configuración de la historia de la nación venezolana.

\section{Modernidad/Colonialidad/Decolonialidad como nueva visión sociocrítica decolonial}

Muchos de los argumentos considerados desde los referentes teórico-conceptuales, pasan a formar parte de una problemática que cambia a cada instante; pero que sus raíces permanecen en las críticas de un mundo de incertidumbres, donde la subjetividad e intersubjetividad parecieran inseparables de aquello que se observa y escribe. Esta situación sirve un poco para interpretar la diversidad como un obstáculo en el momento de sistematizar experiencias vividas que, en lo sucesivo, se convirtieron en el camino para ver la orientación que se puede dar en relación con el objeto de estudio. En este caso, la situación problemática, en continua interacción teóricoempírica, y la cual conduce a abordar la perspectiva teórica Modernidad/Colonialidad/ Decolonialidad, en relación con la experiencia educativa del CEICO en el pueblo de Cuyagua y la forma cómo se dieron las interacciones con los sujetos de la investigación, como una propuesta de construcción desde el punto de vista cualitativo. Como bien se sabe, el tema de la investigación está centrado en la educación integral comunitaria, a través de la experiencia del CEICO de Cuyagua, el cual conduce a la mirada ontológica-epistemológica que, más allá de lo meramente filosófico, y ello es el espacio buscado y guiado en todo momento por un proceso de investigación con la gente.

La perspectiva teórica Modernidad/Colonialidad/Decolonialidad es así un proyecto y una apuesta epistémico política que surge del encuentro no lineal, ni sintético, de múltiples miradas y el cual proporciona respuestas alternativas a las preguntas que evidencian las limitaciones de la teoría crítica moderna.

Sus raíces están ancladas en un abanico que va desde la teología de la liberación y la filosofía de la liberación de Enrique Dussel, pasando por la teoría de la dependencia, los grandes aportes del 
pensamiento latinoamericano, la semiología y la teoría del sistema-mundo de Immanuel Wallerstein, pero que además representa un giro, el cual pone en juicio la forma establecida de legitimar el conocimiento, desde los paradigmas de investigación social, tanto tradicional y hegemónico y el positivista, así como los emergentes que, para el caso del sociocrítico, se encuentran ligados a la teoría crítica de la Escuela de Frankfurt, fiel representante del eurocentrismo, aquí cuestionado y el cual aparece siempre en conflicto en las reflexiones de los problemas latinoamericanos.

De la misma forma, esta perspectiva teórica corresponde al pensamiento de un conjunto de intelectuales de distintas procedencias, principalmente latinoamericanos, como Edgardo Lander (venezolano), Arturo Escobar (colombiano) y Aníbal Quijano (peruano), recientemente fallecido, entre otros, quienes hacen una relectura crítica de la modernidad y crean una teoría crítica decolonial, la cual va más allá de la crítica eurocéntrica de la Escuela de Frankfurt, Alemania y en la cual se fundamenta el paradigma sociocrítico, a partir de lo que ellos consideran el giro decolonial como propuesta político-epistémica.

Cabe aclarar que la perspectiva Modernidad/Colonialidad/Decolonialidad no es ni un paradigma de investigación social, ni representa una ruptura paradigmática, en términos tradicionalmente kuhnianos, de cambiar un paradigma por otro, pues estas dos visiones se corresponden con la ciencia occidental, moderna, por supuesto eurocéntrica, en profunda y generalizada crisis, tanto en las ciencias sociales como en las naturales, las cuales pretenden dar soluciones totales y únicas a la complejidad del mundo social y físico además, sin considerar, por lo menos, que la del mundo de los seres humanos y sus relaciones sociales es mayor que la del mismo universo.

De lo que se trata, en esencia, es de reconocer como en el presente se está viendo y viviendo nuevas emancipaciones, ya no de los movimientos sociales clásicos del siglo XX y que, por ende, se requieren de nuevas maneras de comprensión, esto es, de ver el Sur desde el Sur y no solamente desde el Norte (Europa y los Estados Unidos) como se ha venido haciendo tradicionalmente.

En este sentido, la perspectiva teórica, en la cual se ubica el autor, no puede separarse del eurocentrismo del colonialismo, porque ambos han estado presentes en la configuración de las ciencias sociales en todo el mundo.

Una mejor argumentación se encuentra en esta reflexión de Lander (2000):

El eurocentrismo y el colonialismo son como cebollas de múltiples capas. En diferentes momentos históricos del pensamiento social crítico latinoamericano se han develado algunas de estas capas. Posteriormente, siempre ha sido posible reconocer aspectos y dimensiones (nuevas capas de ocultamiento) que no habían sido identificadas por las críticas anteriores. 
Cuando se profundiza en lo denominado como "teoría crítica", se encuentra que esto corresponde a una visión eurocéntrica, la cual poco dice de las realidades de cada uno, por eso es necesario superarla, a partir de una nueva visión sociocrítica decolonial, fundamentada desde la perspectiva teórica Modernidad/Colonialidad/Decolonialidad, y que parece más pertinente para adoptar, desde los propios movimientos sociales, con sus epistemologías, desde los diversos conocimientos situados en los distintos contextos latinoamericanos y caribeños en la actualidad y en contra del racismo, el eurocentrismo y el colonialismo en todos sus niveles.

Esta perspectiva teórica ha sido para el autor y, sin lugar a dudas, la más apropiada para abordar la temática, la problemática y el objeto de estudio de la investigación, porque las toca de manera directa y profunda, sobre todo cuando se refiere al racismo epistemológico, porque es el mayor nivel de racismo al que se puede llegar desde el paradigma "científico" del pensamiento único, unidimensional, el cual le otorga el derecho de validarse a sí mismo y de desconocer a todo lo que no se le parezca.

\section{Afroepistemología y epistemología del Sur}

El proceso de investigación, por tanto, llevó al autor a relacionar la categoría de afroepistemología como epistemología decolonial y como construcción de conocimiento nuevo y la teoría de la epistemología del Sur, aunque los autores de dichos conceptos tengan orígenes culturales y académicos diferentes puesto que: un afrovenezolano y un portugués, ambos comparten la preocupación por la visibilización de los conocimientos de grupos que están invisibilizados, (García, 2006 y 2010) -por los de los africanos y sus descendientes-, mientras Santos (2009), con una visión más global, reconoce que en el mundo existen clases, pueblos y grupos sociales víctimas del colonialismo/capitalismo y de la colonialidad del poder, del saber, del ser y de la naturaleza, cuyas prácticas no son visibles y creíbles para el eurocentrismo, de allí que se proponga todo lo contrario:

La búsqueda de conocimientos y de criterios de validez del conocimiento, que otorguen visibilidad y credibilidad a las prácticas cognitivas de las clases, de los pueblos y de los grupos sociales que han sido históricamente victimizados, explotados y oprimidos, por el colonialismo y el capitalismo globales. El Sur es, pues, usado aquí como metáfora del sufrimiento humano sistemáticamente causado por el colonialismo y el capitalismo.

Para Santos (2009), los conocimientos significan epistemologías diversas y argumenta que la epistemología hegemónica se encuentra en crisis debido, entre otras causas, a su visión lineal y fragmentaria del mundo, por ello aboga por reconocer y validar, lo cual es hacer justicia cognitiva y lo que contribuiría también a la justicia social en nuestro planeta. 
La afroepistemología es una propuesta epistémica y política descolonizadora, decolonial y liberadora, como base para una educación en ese mismo sentido, con la pedagogía del cimarronaje como práctica, los cuales se unen para ser fundamentos de la pedagogía y las otras epistemologías decoloniales, donde la afroepistemetódica contribuye a reconstruir los conocimientos de la diáspora africana desde adentro, considerando las historias, maneras de ser y de saber de las(os) afrodescendientes.

Es importante que la afroepistemología se reconozca y se inscriba explícitamente dentro de las epistemologías del Sur, en las otras epistemologías, críticas, no eurocéntricas y que dialogue profundamente con epistemologías como la indígena, la feminista, la musulmana, entre otras, para hacer un frente común contra la epistemología positivista, la cual pretende erigirse en patrón de validez y cientificidad, fundamento del sistema capitalista, que históricamente ha violentado a muchos grupos, pueblos y clases sociales alrededor del mundo.

\section{Conclusiones}

Destacar la posibilidad de realizar una investigación con un movimiento social, a partir de una temática y una problemática desde su necesidad e interés, descartando de entrada la posibilidad de ser "objetivos" y "neutrales", algo que, por demás, no se pretende en la investigación cualitativa. Pero sí con la rigurosidad y la reflexividad, desde una postura política crítica; pues todo ello se convirtió en una gran oportunidad para acercarse a la comprensión de una experiencia educativa y pedagógica muy significativa para la ROA y que, con esa visión, sería muy interesante abordar otras experiencias de ese tipo, en otros contextos interculturales desde las epistemologías y pedagogías otras y decoloniales.

Aun cuando esta investigación surge desde una visión cualitativa, pero en perspectiva decolonial, debido al tema y objeto de estudio construido, es que se hace necesario ir más allá del paradigma de investigación social emergente sociocrítico, el cual está basado en la teoría crítica de la Escuela de Frankfurt, sin dejar de reconocer sus aportes a la teoría social, y de la cual hay que admitir que es fundamentalmente eurocéntrica y colonial, por lo cual se tuvo que optar por reconstruir la perspectiva teórica Modernidad/Colonialidad/Decolonialidad como una nueva visión sociocrítica decolonial, mucho más acorde con las transformaciones que se tienen curso en este siglo en América Latina y el Caribe y en el Sur global, además, porque recoge lo mejor del pensamiento latinoamericano, como parte de una mirada fundamental para comprender una experiencia educativa y pedagógica, similar a la vivida en el pueblo de Cuyagua. 
En relación con la educación integral comunitaria, combinada con la afroepistemología y afroepistemetódica, se pudo ver como esta propuesta educativa y pedagógica se convirtió en una verdadera alternativa a la educación tradicional, bancaria, en el sentido freiriano, una basada en el racismo epistémico donde, por ejemplo, solo se reconoció a la universidad (moderna, eurocéntrica, hegemónica y positivista) y por ello se convirtió en el único lugar válido para la producción de conocimiento, esto en contra de una educación propia, contextualizada, pertinente y descolonizada, la cual visibiliza a la comunidad, desde la pedagogía de la oralidad, con su inmensa sabiduría ancestral y para el autor, como una de las figuras más relevantes. A esta visión se suman las(os) maestras(os) del pueblo quienes, sin título académico pero con mucha conciencia, experiencia y conocimientos ancestrales, transmitidos de boca a oído, de generación en generación, participaron en igualdad de condiciones con las(os) profesoras(es) en el proceso de enseñanza y aprendizaje del centro educativo en lo local.

La afroepistemología y afroepistemetódica como epistemología propia, reivindicada por la ROA, debido a su carácter crítico y decolonial y como categoría en construcción desde adentro, basada en el conocimiento y la investigación, situada dentro de una geopolítica, constituyen una episteme y una pedagogía cimarrona, la cual se propone sea incorporada en todos los currículos del país, no solamente desde la Cátedra Libre África, porque contiene aportes muy valiosos para reconstruir la historia de la nación venezolana, desde la perspectiva afro, con sus grandes aportes morales y políticos, en tiempos de revolución, como una propuesta alternativa a la epistemología positivista, la cual se considera legítima, válida, la única y la científica, que es producto de la colonialidad del saber, del poder, del ser y de la naturaleza.

Por lo anterior se considera imperativo que las prácticas, conocimientos y saberes de las comunidades afrodescendientes, se reconozcan al mismo nivel de importancia y legitimidad otorgada al conocimiento académico, inclusive el doctoral, producido en las universidades tradicionales y, más aún, en las creadas por la Revolución en las que urge romper esa jerarquización, impuesta históricamente, que las mantiene invisibilizadas, producto del racismo epistémico.

Queda claro, que aunque se argumente lo opuesto, no existe una sola vía para producir conocimientos, existe en el mundo una pluriculturalidad y diversidad epistémica, al igual que en la educación y la pedagogía, y ésta no se limita a la proporcionada por el sistema educativo oficial.

Las comunidades afrodescendientes y sus organizaciones, desde hace tiempo, han dejado de ser objeto de estudio de las(os) investigadoras(es) positivistas y se han vuelto protagonistas de sus propios procesos de investigación acerca de sus propias realidades, han creado su propia escuela 
de pensamiento y su visión de la historia, además son fundamentalmente sujetos políticos e intelectuales que, para fundamentar la experiencia educativa y pedagógica de Cuyagua, por ejemplo, han hecho uso de su afroepistemología y afroepistemetódica y del método de la Investigación-Acción de manera profunda y reflexiva.

Con mucha determinación, es el momento de trascender la educación tradicional en el país, esa donde el saber es fragmentado y disciplinario, descontextualizado y limitado a la reproducción del capital y la globalización neoliberal, la misma que reproduce el racismo epistémico con sus funestas consecuencias en la subjetividad de las(os) estudiantes, dando como resultado la colonización de las mentes. Por el contrario, se busca una educación que, desde la primaria y hasta el doctorado, sea política, crítica, liberadora y descolonizada, que emancipe y no discrimine, pero que además identifique el entorno y su contexto histórico, social, económico, cultural, político y geográfico, con pertinencia y pertenencia desde lo local, de acuerdo con las características específicas de la población.

\section{Referencias}

Centro de Estudios Latinoamericanos "Rómulo Gallegos" (2013) Convocatoria al Primer Encuentro "Visibilizando a los pueblos indígenas y afrodescendientes: por una estrategia para la erradicación de la discriminación, el neocolonialismo y el racismo en Venezuela". Caracas, febrero 22 de 2013.

Fals Borda, Orlando y González, Raquel. (1991). Acción y conocimiento: Cómo romper el monopolio con investigación-acción participativa. Santafé de Bogotá: Cinep.

García, Jesús. (2006). Caribeñidad: Afroespiritualidad y afroepistemología. Caracas: El perro y la rana.

García, Jesús. (2010). Afroepistemología y afroepistemetódica. En Sheila Walker S. (Comp.). Conocimiento desde adentro: Los afrosudamericanos hablan de sus pueblos y sus historias: Tomo I (pp. 69 - 87). Bolivia: Programa de Investigación Estratégica (PIEB).

González Rey, Fernando Luis. (2007). Investigación cualitativa y subjetividad: Los procesos de construcción de la información. México: McGraw-Hill.

Grosfoguel, Ramón. (2011). La descolonización del conocimiento: Diálogo crítico entre la visión descolonial de Frantz Fanon y la sociología descolonial de Boaventura de Sousa Santos. En Formas - Otras: Saber, nombrar, narrar, hacer. IV Training Seminar de jóvenes 
investigadores en Dinámicas Interculturales [Libro en línea]. CIDOB Edicions, Barcelona. Disponible:http://www.cidob.org/es/publicaciones/monografias/monografias/formas_otras_ saber_nombrar_narrar_hacer[Consulta: 2012, Octubre 23].

Herrera Salas, Jesús María. (2009). Economía política del racismo en Venezuela. Caracas: Mihail Batjin.

Ibáñez Gracia, Tomás. (1990). Aproximaciones a la psicología social. Barcelona: Sendai.

Lander, Edgardo. (Comp.) (2000). La colonialidad del saber: eurocentrismo y ciencias sociales. Perspectivas Latinoamericanas. Buenos Aires: CLACSO.

Martínez de Crespo, María Mercedes. (2003). Compilación del CEICO, Centro Educativo Integral Comunitario (CEICO) Cuyagua. Presentación en Power Point. Cuyagua, estado Aragua.

Ministerio del Poder Popular para la Educación, Unidad Educativa Nacional "Cuyagua”, estado Aragua. (2008). Proyecto institucional del Centro Educativo Integral Comunitario Cuyagua, CEICO Cuyagua. Maracay: Autor.

Quijano, Aníbal. (2009). Colonialidad del poder y clasificación social. En Ramón Grosfoguel y José Romero Lossaco (Comps.). Pensar decolonial. Caracas: La Urbana.

Santos, Boaventura de Sousa. (2009). Una epistemología del sur: la reinvención del conocimiento y la emancipación social. México: Siglo XXI-CLACSO.

Valles, Miguel S. (1999). Técnicas cualitativas de investigación social. Reflexión metodológica y práctica profesional. Madrid: Síntesis.

\section{Derechos de autor}

Los derechos de autor de este artículo son retenidos por los autores, con los derechos de primera publicación otorgados a la revista. Este es un artículo de acceso abierto distribuido bajo los términos y condiciones de la licencia Creative Commons: Reconocimiento-NoComercialCompartirIgual 4.0 Internacional 
Derecho y Realidad (2017) 15(30): e28175-1

ISSN 1692-3936 ISSN-L 2619-5607

Artículo de investigación

Derecho real

Julio - Diciembre 2017 